\title{
Desarrollo vegetativo y fenología del mamón (Carica papaya $L$.) bajo dos condiciones de cultivo
}

\author{
Collavino, Agostina A. ; Florentin, Jorge L.; Gaiad, José E. ${ }^{2}$; Alayón Luaces, Paula ${ }^{2}$ \\ ${ }^{1}$ Ingenieros Agrónomos; ${ }^{2}$ Docentes de Fruticultura, Facultad de Ciencias Agrarias Universidad \\ Nacional del Nordeste. Sargento Cabral 2131 (3400) Corrientes Argentina. \\ E-mail: palayonluaces@yahoo.com
}

\begin{abstract}
Resumen
La región NEA cuenta con zonas aptas para el cultivo de mamón, sin embargo las condiciones durante el invierno hacen que estos cultivos sean destinados principalmente a la industria. Las bajas temperaturas y más aún las heladas, son los principales factores limitantes para la producción de este cultivo en zonas subtropicales. Sin embargo en el NEA y en Corrientes en particular, el uso de invernaderos favorecería el desarrollo de este cultivo. Las condiciones micro-meteorológicas en este tipo de sistemas de producción se modifican por el forzado traduciéndose estas modificaciones en un comportamiento diferente del cultivo comparado con su producción al aire libre. El objetivo de este trabajo fue evaluar el desarrollo vegetativo y la fenología del cultivo de mamón bajo dos condiciones de cultivo, en invernadero y al aire libre. Se realizó la comparación entre dos tratamientos de mamón cv. Formosa: uno en condiciones de aire libre y otro en invernadero. Se midió el crecimiento vegetativo a través de la: Altura de planta; Número de hojas; Número de nudos; Volumen de copa; Diámetro de tronco y se realizó el seguimiento fenológico. Las condiciones de cultivo bajo invernadero favorecieron el desarrollo vegetativo, principalmente en altura de planta y volumen de copa en comparación con el cultivo al aire libre. La entrada en producción en condiciones de invernadero fue antes de los meses de menor temperatura. Las plantas que crecieron al aire libre presentaron un periodo desde floración a cuajado de frutos más extenso que aquellas cultivadas en invernadero, afectando la entrada en producción coincidente con los meses más fríos del año.
\end{abstract}

Palabras clave: papaya, cultivo tropical, invernaderos, crecimiento.

\begin{abstract}
In the Northeast region of Argentina (NEA) there are suitable zones for papaya crop, however winter conditions made that fruit destination be the industry. Low temperatures and even more the frost, are the principal limitations for papaya crop in subtropical region. However in NEA, and moreover at Corrientes, there are many greenhouse structures that could be used for papaya production. Micrometeorological condition in this type of production could influence on crop behavior compared with outside production. The aim of this work was to evaluate the vegetative development and phenology of papaya plant under two growth conditions, greenhouse and field cultivation. Two experimental plots were made using cultivar Formosa: under field cultivation and the other under greenhouse conditions. Vegetative growth was measured through plant height, number of leaves, number of nodes, canopy volume and trunk diameter. Phenological stage was monitored. Cultivation under greenhouse conditions improved vegetative growth, mainly in plant height and canopy volume compared to field conditions. Under greenhouse conditions, plants begin its production before the cooler months. Under field conditions plants had a more extensive period from flowering to fruit set that coincides with the coldest months of the year affecting the productivity.
\end{abstract}

Key words: papaya, tropical crop, greenhouse, growth.

\section{INTRODUCCIÓN}

Carica papaya L., comúnmente conocida como 'mamón', 'papaya', 'mamao', 'lechosa', pertenece al orden Parietales, familia Caricaceae, es una planta arborescente perennifolia, cuya copa es abierta y redondeada y las hojas son simples de pecíolo largo.

Recibido: 13/07/2015. Aceptado: 10/12/2015
Los principales países productores de mamón son India, Brasil y Nigeria los cuales proveen el $71 \%$ de la producción mundial. En nuestro país, hasta hace tres años la producción de mamón alcanzó los 2000 Mg (FAOSTAT, 2012) siendo Salta y Jujuy las principales provincias productoras. Sin embargo en las provincias del NEA, comprendidas por Corrientes, Misiones y Formosa existen sectores productivos 
asociados a pequeñas empresas. Según Parra (2012) en el año 2010 en Argentina se importaron $889 \mathrm{Mg}$ de fruta de mamón, y en el año 2011 ingresaron al país unas $459 \mathrm{Mg}$, lo cual es indicativo de una demanda insatisfecha con la producción nacional.

El fruto del mamón es valorado para su consumo en fresco por su sabor y por su alto valor nutritivo y aporte de vitaminas y minerales (Alonso et al., 2008). Sin embargo también puede ser aprovechado con fines industriales y medicinales para extracción de papaína, una enzima proteolítica muy apreciada para ablandar carnes y para aclarar bebidas, como así también en industrias alimenticias para la producción de dulces, jaleas y frutos abrillantados.

Por ser de origen tropical las condiciones agroecológicas más favorables para su desarrollo y crecimiento son aquellas caracterizadas por alta radiación solar, ausencia de heladas y pluviometrías de alrededor de $1300 \mathrm{~mm}$ anuales (Samson, 1991).

La región del NEA cuenta con zonas aptas para el cultivo de mamón, sin embargo las condiciones durante el invierno determinan que estos cultivos sean destinados principalmente a la industria. Esto es debido a que para el aprovechamiento de los frutos para su consumo en fresco los mismos deben alcanzar su madurez organoléptica apropiada, lo que representa que la cosecha se realice en los meses de abril y mayo, dificultándose la obtención de fruta de calidad debido a las bajas temperaturas.

Según lo citado por Knight (1980) el óptimo desarrollo y crecimiento del mamón se obtiene entre $21^{\circ}$ y $33^{\circ} \mathrm{C}$, clasificándose a este cultivo como una especie tropical sensible a las bajas temperaturas (Ogden et al., 1981). Cuando las temperaturas descienden por debajo del óptimo y según sea el momento del ciclo del cultivo se pueden presentar anomalías. GalánSaúco y Rodríguez-Pastor (2007) citan que temperaturas por debajo de los $18^{\circ} \mathrm{C}$ provocan carpeloidia de los estambres (frutos conocidos como 'cara de gato' sin valor comercial), cambios de género de la flor, reducción de la viabilidad del polen y menor contenido de azúcares totales en frutos.

Las bajas temperaturas y más aún las heladas, son los principales factores limitantes para la producción de mamón en zonas subtropicales. Sin embargo en el NEA y en Corrientes en particular, el uso de invernaderos existentes favorecería al desarrollo de este cultivo. Esta posibilidad no solo se traduce en beneficios económicos, sino que también posibilita la diversificación de cultivos frutales y el aprovechamiento de estas estructuras de forzado, ya que la producción de frutales en la provincia se limita básicamente a los cítricos. Por una parte Corrientes es la provincia con mayor superficie con invernaderos del NEA, existiendo cerca de 1000 ha, de las cuales el 65\% están dedicadas al tomate y el $29 \%$ al pimiento (Lenscak y Mansutti, 2009).

Las condiciones climáticas que suceden en los sistemas de forzado se modifican en comparación al aire libre, traduciéndose en un comportamiento diferente del cultivo. El objetivo de este trabajo fue evaluar el desarrollo vegetativo y la fenología del cultivo de mamón bajo dos condiciones de cultivo: en invernadero y al aire libre.

\section{MATERIALES Y MÉTODOS}

El trabajo se realizó en Santa Ana, departamento San Cosme, Corrientes (27 $\left.26^{\prime} \mathrm{S}-58^{\circ} 36^{\prime} \mathrm{O}\right)$, Argentina. El experimento consistió en realizar una comparación entre un cultivo de mamón al aire libre y otro en invernadero. Se utilizó un invernadero tipo capilla con ventilación cenital de $24 \mathrm{~m}$ x $14 \mathrm{~m}$, de madera, con laterales de $2,5 \mathrm{~m}$ de altura y $3,5 \mathrm{~m}$ de máxima altura con ventilación cenital de $0,50 \mathrm{~m}$, con una superficie experimental de $336 \mathrm{~m}^{2}$ y en un sector al aire libre de $12 \mathrm{~m} \times 18 \mathrm{~m}\left(216 \mathrm{~m}^{2}\right)$, cercano al invernadero.

$\mathrm{El}$ material vegetal utilizado fueron plantas de $\mathrm{Ca}$ rica papaya L. 'Formosa'.

La plantación se realizó el 10 de octubre, la cual en condiciones de aire libre natural se ejecutó sin labranza a un distanciamiento de $1,5 \mathrm{~m}$ entre plantas y 1,5 $s$ entre líneos (4444 plantas ha ${ }^{-1}$ ). En el invernadero las plantas se ubicaron en un marco de plantación de 1 metro entre plantas y 1,5 metros entre lineo (6666 plantas ha ${ }^{-1}$ ). En ambos tratamientos al momento de la plantación se colocaron $3 \mathrm{Kg}$ de estiércol vacuno estacionado en el hoyo, para facilitar el enraizamiento y lograr un buen arraigue de los plantines.

El diseño experimental fue en Bloques Completos al Azar (factor de bloqueo pendiente de 0,5\%). Se realizaron parcelas experimentales formadas por 10 plantas siendo 2 borduras y 8 las plantas centrales útiles evaluadas, con cuatro repeticiones

Para analizar la evolución de las plantas se realizaron mediciones de crecimiento y desarrollo. Se examinaron el total de plantas por tratamiento (32 plantas por tratamiento) y en las mismas se determinó:

Altura de planta (cm): esta medida se tomó desde la base del tallo hasta el ápice.

Número de hojas: se contaron todas las hojas por planta.

Número de nudos: se contaron todos los nudos desde las cicatrices de la base hasta el nudo de la última hoja desplegada.

Volumen de copa $\left(\mathrm{cm}^{3}\right)$ : para determinarlo se midió la proyección de la copa (diámetro 1 y diámetro 2) y la altura de la misma, para luego calcularlo [diámetro1 $\mathrm{x}$ diámetro2 $\mathrm{x}$ altura de copa].

Diámetro de tronco $(\mathrm{cm})$ : con calibre se midió el diámetro de tallo a $10 \mathrm{~cm}$ del suelo. 
Seguimiento fenológico: se observó el desarrollo de la planta tomando registro de los estadios en los cuales se encontraba cada planta. Los registros fenológicos comenzaron a tomarse en el momento que se visualizaron los primeros pimpollos (inicio de floración). Se consideró inicio de fase cuando el $50 \%$ de los individuos se encontraban en dicho momento.

Altura de inserción primera flor funcional $(\mathrm{cm})$ : esta medida se tomó desde la base del tallo hasta donde se encontraba la primera flor funcional.

Las variables vegetativas se midieron hasta plena floración (cuando el $50 \%$ de las plantas del lote se encontraba en floración).

El análisis estadístico de los datos se realizó utilizando el software InfoStat versión 2013 (Di Rienzo et al., 2012). Previo a realizar las comparaciones de las variables medidas se probaron los supuestos de normalidad de los datos (Test de Shapiro-Wills) y homogeneidad de varianza. Posteriormente se realizaron análisis de la varianza (ANOVA) y comparación de medias utilizando test de Tuckey $(\mathrm{p}<0,05)$.

\section{RESULTADOS Y DISCUSIÓN}

Las temperaturas medias mensuales registradas en el mes de plantación fueron $4,3^{\circ} \mathrm{C}$ superiores en el invernadero que aquellas registradas al aire libre (Tabla 1). Nakasone y Paull (1998) señalan que el ran go de temperaturas para un buen crecimiento de este cultivo es de 15 a $35{ }^{\circ} \mathrm{C}$, con un rango de tempera turas óptimas para la fotosíntesis de 25 a $30{ }^{\circ} \mathrm{C}$. La temperatura mínima para un crecimiento satisfacto rio es $15{ }^{\circ} \mathrm{C}$, pues temperaturas inferiores inhiben el desarrollo de las flores. A $0{ }^{\circ} \mathrm{C}$ se presentan fuertes daños al follaje; a $-2{ }^{\circ} \mathrm{C}$ los frutos se dañan conside rablemente y a $-4^{\circ} \mathrm{C}$ la planta muere.

Si bien en ambos sistemas de cultivo la temperatura media se encuentra por encima de la base del cultivo $\left(15^{\circ} \mathrm{C}\right)$ las temperaturas diarias al aire libre fueron más bajas durante algunas horas del días que aque-

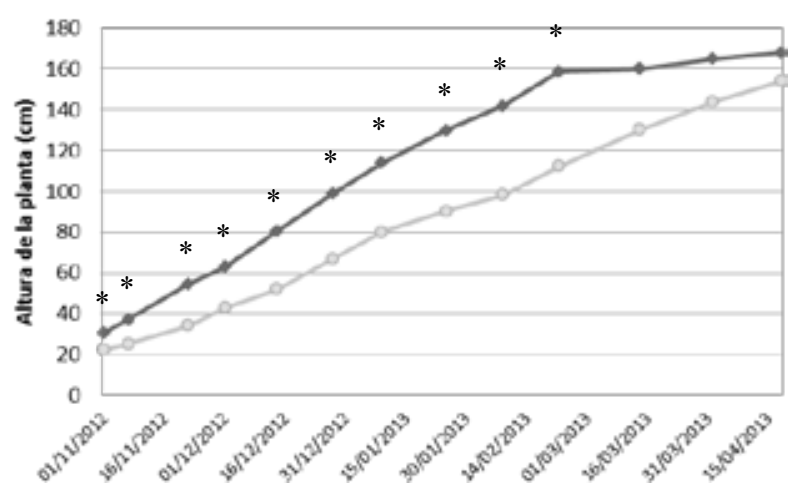

Figura 1. Altura promedio de plantas de mamón (Carica papaya L.) en dos sistemas de cultivo ( $\diamond)$ : invernáculo; (○) aire libre; * presentan diferencias significativas $(\mathrm{p} \leq 0,05)$. llas registradas en el invernadero, esta diferencia fue la que incidió en que el crecimiento de las plantas en invernadero no se detenga luego del trasplante.

La altura de las plantas durante los primeros 5 meses de cultivo fueron significativamente más altas $(\mathrm{P} \leq 0,05)$ que aquellas medidas en el cultivo al aire libre.

Asociado a este parámetro, se analizó la cantidad de nudos en el tallo principal, observándose que durante los primeros cuatro meses a partir de la plantación no hubo diferencias $(\mathrm{P} \leq 0,05)$ entre las plantas en invernadero y al aire libre (Fig. 2). Las diferencias recién se encontraron luego de los 5 meses de cultivo, indicando que la altura en las plantas cultivadas en invernadero se debió a que los entrenudos eran más largos ya que la cantidad respecto al aire libre eran iguales durante ese periodo. El alargamiento de los entrenudos está asociado a la cantidad y calidad de luz (Campostrini y Glenn, 2007), y en este sentido en el invernadero se registraron valores de radiación fotosintéticamente activa incidente un $25 \%$ menores

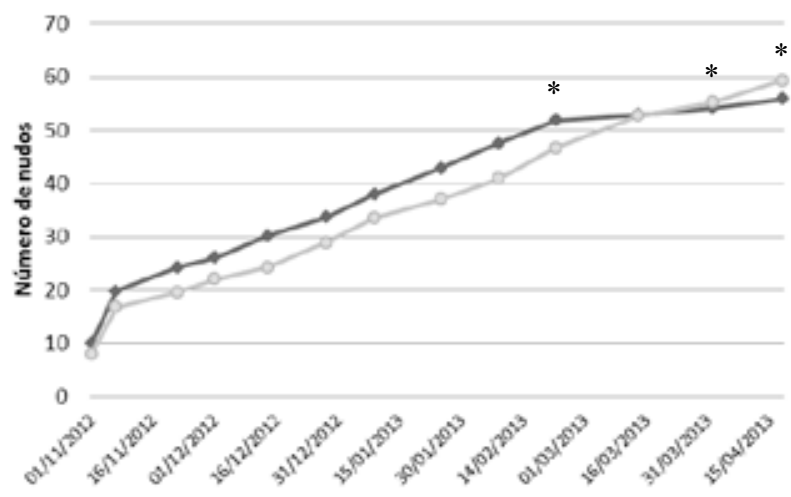

Figura 2. Número de nudos promedio de plantas de mamón (Carica papaya L.) en dos sistemas de cultivo ( $\diamond$ ): invernáculo; (○) aire libre; * presentan diferencias significativas $(\mathrm{p} \leq 0,05)$.

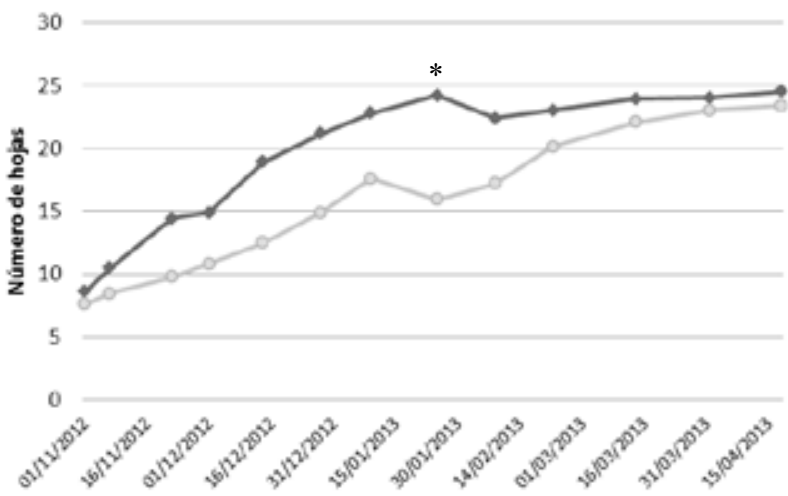

Figura 3. Número de hojas promedio de plantas de mamón (Carica papaya L.) en dos sistemas de cultivo (४): invernáculo; (O) aire libre; * presentan diferencias significativas $(\mathrm{p} \leq 0,05)$. 


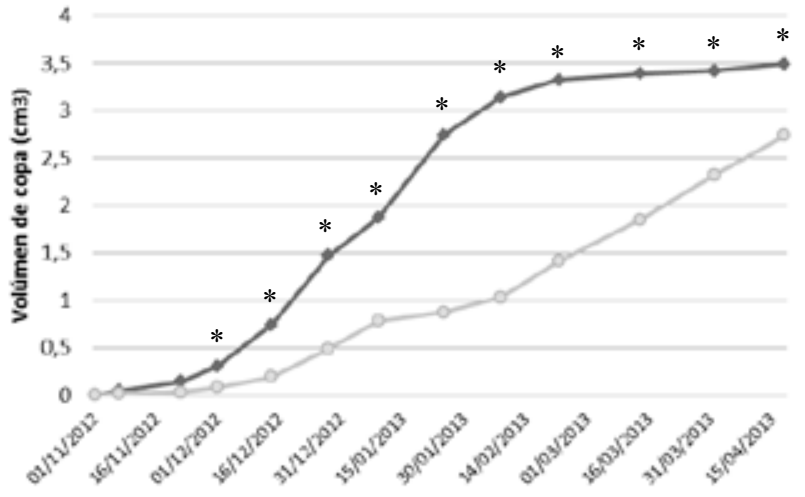

Figura 4 . Volumen de copa promedio de plantas de mamón (Carica papaya L.) en dos sistemas de cultivo $(\diamond)$ : invernáculo; $(\odot)$ aire libre; * presentan diferencias significativas $(\mathrm{p} \leq 0,05)$.

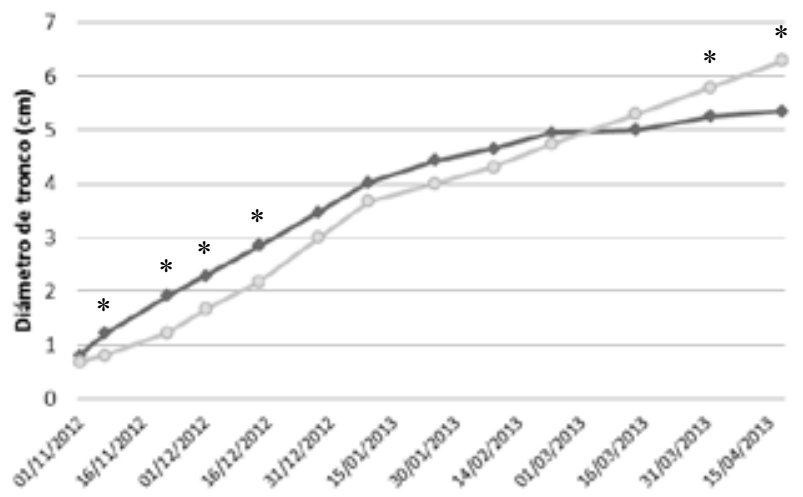

Figura 5. Diámetro de tronco promedio de plantas de mamón (Carica papaya L.) en dos sistemas de cultivo $(\diamond)$ : invernáculo; $(\circ)$ aire libre; * presentan diferencias significativas $(\mathrm{p} \leq 0,05)$.

que aquellas registradas al aire libre durante todo el ciclo evaluado.

La cantidad de hojas desarrolladas en ambos sistemas de cultivo fue similar durante todo el ciclo estudiado, diferenciándose únicamente a mediados de febrero. Las altas temperaturas registradas en invernadero afectaron negativamente a las plantas en este periodo del año (Fig. 3), ya que a partir de febrero las plantas cultivadas en invernadero ya no aumentaron la cantidad de hojas, las cuales se mantuvieron y solo se terminaron de desarrollar aquellas que ya se habían diferenciado anteriormente, sin embargo en las plantas que fueron cultivadas al aire libre esta estabilización recién se manifestó en marzo.

Las plantas que crecieron en el invernadero presentaron un acelerado incremento en el volumen de copa durante los primeros tres meses (Fig. 4). El mayor volumen de copa se mantuvo prácticamente durante todo el ciclo de crecimiento, aunque se observó una tendencia a igualarse recién al final del ciclo en el mes de marzo donde ya no se encontraron diferencias $(\mathrm{P} \leq 0,05)$ entre ambos sistemas de cultivo.
Tabla 1. Temperaturas medias mensuales $\left({ }^{\circ} \mathrm{C}\right)$ registradas durante el ciclo productivo del cultivo de "Mamón" Carica papaya L. 'Formosa', en condiciones cultivo al aire libre y en invernadero, en Santa Ana, Corrientes Argentina.

\begin{tabular}{lcc}
\hline MESES & AIRE LIBRE & INVERNÁCULO \\
\hline Octubre & 21,012 & 25,374 \\
Noviembre & 25,750 & 31,683 \\
Diciembre & 28,139 & 31,490 \\
Enero & 27,431 & 31,109 \\
Febrero & 26,509 & 30,560 \\
Marzo & 23,200 & 27,880 \\
Abril & 20,071 & 24,000 \\
\hline
\end{tabular}

Esta diferencia medidas en el volumen del dosel podría atribuirse al mayor tamaño de las hojas en las plantas que crecieron en el invernadero, dado que si se considera la cantidad de hojas, según se observó prácticamente no hubo diferencias entre sistemas de cultivo (Fig. 3). Este incremento en la expansión foliar, probablemente este asociado a las temperaturas más favorables ocurridas en el invernadero las cuales siempre estuvieron por encima de aquellas registradas al aire libre (Tabla 1). Los resultados medidos del mayor desarrollo de copa y altura de las plantas en el invernadero, coincide con los citado por Vázquez García et al., 2008; quienes encontraron una correlación positiva entre la acumulación de unidades de calor y estas dos variables estudiadas. Por otra parte, posiblemente no sea sólo atribuida a la temperatura las diferencias observadas, ya que el viento puede incidir negativamente en el desarrollo de las hojas (Marler, 2011). Por lo tanto, y aunque no fue medido en este trabajo, el menor régimen eólico en el interior de los invernaderos, podría también haber incrementado el desarrollo de volumen de copa de las plantas en comparación con las cultivadas al aire libre.

El diámetro de tronco aumentó en ambos sistemas de cultivo durante todo el ciclo analizado (Fig. 5), este parámetro en cierto modo indica el vigor de las plantas ( $>$ diámetro $>$ vigor), el cual podría ser considerado como un valor positivo a la hora de selección de la variedad (Rodríguez y Rosell, 2005). Las diferencias se manifestaron hasta fines de diciembre donde en ambos sistemas las plantas siguieron engrosando el tronco sin presentar diferencias entre sistemas de cultivo. Este comportamiento se modifica luego del mes de marzo, donde las plantas que crecieron al aire libre resultaron con un mayor diámetro de tronco en comparación con las plantas cultivadas en el invernadero (Fig. 5).

En las Tablas 2 y 3 se presentan las mediciones fenológicas realizadas. Se observó que el inicio de floración fue a partir del 9 de enero independientemente del sistema de cultivo, por lo que el periodo de desarrollo vegetativo fue de 91 días coincidente con 
Tabla 2. Seguimiento fenológico del cultivo de "Mamón" Carica papaya L. 'Formosa', en condiciones de cultivo en invernadero plástica.

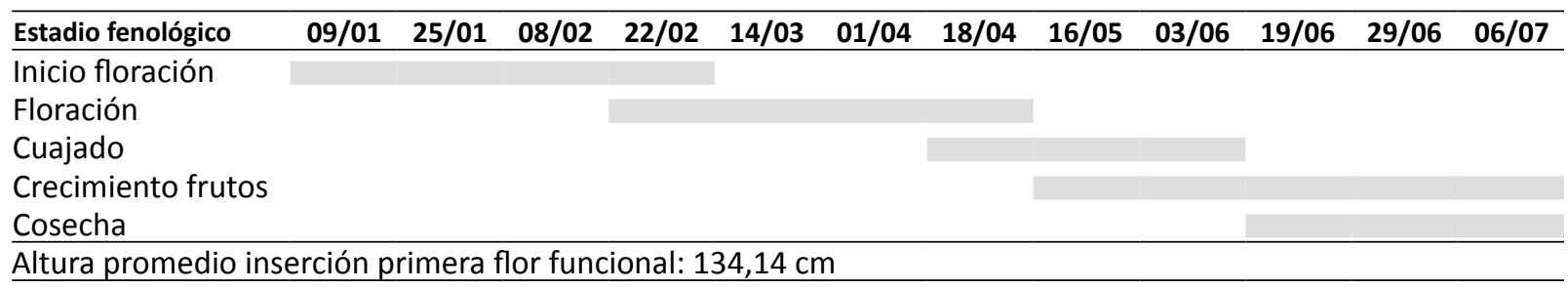

Tabla 3. Seguimiento fenológico del cultivo de "Mamón" Carica papaya L. 'Formosa', en condiciones cultivo al aire libre.

\begin{tabular}{|c|c|c|c|c|c|c|c|c|c|c|c|c|}
\hline \multirow{2}{*}{\multicolumn{13}{|c|}{$\frac{\text { Estadio fenológico }}{\text { Inicio floración }}$}} \\
\hline & & & & & & & & & & & & \\
\hline Floración & & & & & & & & & & & & \\
\hline Cuajado & & & & & & & & & & & & \\
\hline Crecimiento frutos & & & & & & & & & & & & \\
\hline Cosecha & & & & & & & & & & & $\mathrm{s} / \mathrm{d}$ & $s / d$ \\
\hline
\end{tabular}

s/d: sin datos.

lo encontrado por Ceccotli et al., (2013) en plantas de mamón cultivadas en Santa Fe Argentina (Tablas 2 y 3). Este período fue de 44 días para las plantas cultivadas en el invernadero más extenso para las plantas cultivadas al aire libre alcanzando los 65 días. Lo cual, considerando la etapa desde floración hasta cuajado de frutos, fueron de 97 y 127 días respectivamente, lo que revela un atraso de 30 días de las plantas de aire libre respecto a las cultivadas en invernadero. La principal diferencia encontrada en el comportamiento fenológico fue que las plantas cultivadas en el invernadero presentaron un solapamiento de los periodos más marcado que en aquellas cultivadas al aire libre. La importancia que reviste estos análisis vinculados con la fenología de la plantas es que permiten entender las respuestas de las mismas a los factores ambientales. También, la diferenciación de las etapas críticas de las plantas, lo cual ayuda a maximizar el uso eficiente de los insumos disponibles, al permitir aplicarlos en dosis óptimas y en el momento en que las plantas lo requieran (Nakasone y Paull, 1998).

Las temperaturas registradas en el invernadero se mantuvieron siempre entre $\operatorname{los} 21^{\circ}$ y $33^{\circ} \mathrm{C}$ (Tabla 1), rango considerado como óptimo para el desarrollo y crecimiento del mamón según lo citado por Knihgt (1980). Sin embargo, al aire libre se midieron temperaturas más bajas, por lo que es probable que la incidencia de las mismas haya sido la causa por la cual las plantas de mamón demoraron más tiempo ( 7 meses desde el momento de plantación) en comenzar a fijar frutos y entrar en producción. Chay-Prove et al. (2000) encontraron que los frutos están listos para ser cosechados de 5 a 6 meses luego del inicio de floración, lo cual coincide con lo observado en las plantas cultivadas en invernadero, no así en las plantas cultivadas al aire libre debido a las bajas temperaturas que se presentaron en los meses de mayo, junio y julio, las cuales detuvieron el desarrollo de los frutos.

En la base de las tablas 2 y 3 se presenta la altura promedio donde se estableció el primer fruto (primera flor funcional). Según Alonso et al. (2008) aquellos cultivares que inicien la primera flor a una altura menor son deseables ya que con ello se facilita la recolección y se disminuyen los costos de la mano de obra. Asimismo, la reducción de la altura de inserción de la primera flor en la papaya, es de gran importancia económica porque permite una mayor longevidad de la cosecha, lo que unido a una fructificación precoz y vigorosidad de la planta, resultan caracteres de interés dentro de las perspectivas del mejoramiento de la papaya (Marin et al., 2006). En este sentido, en las plantas de mamón en invernadero la fijación del primer fruto se dio a una altura promedio de $21,61 \mathrm{~cm}$ superior a aquellas cultivadas al aire libre.

\section{CONCLUSIONES}

Las condiciones del cultivo de mamón en invernadero favorecieron el desarrollo vegetativo, principalmente la altura de planta y volumen de copa, en comparación con el cultivo al aire libre. En el cultivo en invernadero no se observó detención en el crecimiento de las plantas luego del trasplante, lo que se tradujo en un mayor desarrollo vegetativo que permitió alcanzar una cosecha de frutos antes de los meses de menor temperatura. La etapa desde floración hasta cuajado de frutos presentó un atraso de 30 días de las plantas cultivadas al aire libre respecto a aquellas cultivadas en invernadero. Las plantas que crecieron al aire libre presentaron un periodo de floración a 
cuajado de frutos más extenso que aquellas cultivadas en invernadero.

\section{BIBLIOGRAFIA}

Alonso, M.; Tornet, Y.; Ramos, R.; Farrés, E.; Castro, J.; Rodríguez, M.C. 2008. Evaluación de tres cultivares de papaya del grupo solo basada en caracteres de crecimiento y productividad. Cultivos Tropicales 29 (2): 59-64.

Campostrini, E.; Glenn, D.M. 2007. Ecophysiology of papaya: a review. Braz. J. Plant Physiol., 19(4): 413-424.

Céccoli, G.; Panigo, E.S.; Gariglio, N.; Favaro, J.C.; Bouzo, C.A. 2013. Fruit yield and growth parameters of several Carica papaya L. genotypes in a temperate climate. Rev. FCA UNCUYO 45(2): 299-310.

Chay-Prove, P.; Ross, P.; O' Hare, P.; Macleod, N.; Kernot, I.; Evans, D.; Grice, K.; Vawdrey, L.; Richards, N.; Blair, A.; Astridge, D. 2000. Agrilink Series: Your Growing Guide to Better Farming. Paw Paw information Kit. Queensland Horticulture Institute and Department of Primary Industries. $185 \mathrm{p}$.

Di Rienzo, J.A.; Casanoves, F.; Balzarini, M.G.; Gonzalez, L.; Tablada, M.; Robledo, C.W. 2012. InfoStat versión 2012. Grupo InfoStat, FCA, Universidad Nacional de Córdoba, Argentina. URL http://www.infostat.com.ar

FAOSTAT. 2012. http://faostat.fao.org/

Galán-Saúco, V.G.; Rodríguez-Pastor, M.C.R. 2007. Greenhouse cultivation of papaya. Acta Hort. 740:191- 195

Knight, R.J. 1980. Origin and world importance of tropical and subtropical fruit crops. In: Nagy S, Shaw PE (eds), Tropical and Subtropical Fruits: Composition, Properties, and Uses, pp.1-120. AVI Publishing,Westport.
Lenscak,M.P.; Mansutti, J.J. 2009. Relevamiento y Diagnóstico Técnico de las Estructuras de Invernaderos de la Provincia de Corrientes. Publicación EEA Bella Vista. Serie Técnica No 33. 2009. 18 pp.

Marin, S.L.D.; Pereira, M.G.; Amaral Junior, A.T.; Martelleto, L.A.; Ide, C.D. 2006. Heterosis in papaya hybrids from partial diallel of "Solo" and "Formosa" parents. Crop Breeding and Applied Biotechnology 6:24-29.

Marler, T. E. 2011. Growth responses to wind differ among Papaya roots, leaves and stems. HortScience 46: 11051109.

Nakasone, H.; Paull, R. E. 1998. Papaya. In: Tropical Fruits. Crop Production Science in Horticulture. Serie No7. CAB International Walling Ford. pp: 239-269.

Ogden, M.A.H.; Jackson, L.K.; Campbell, C.W. 1981. Florida tropical fruit culture via master gardener. Proc. Fla. Sta. Hort. Soc. 94:222-225.

Parra, P.A. 2012. Papaya o Mamón (Carica papaya) en Argentina. Panorama de la Cadena Agroindustrial Secretaría de Desarrollo Rural y Agricultura Familiar Ministerio de Agricultura, Ganadería y Pesca, Argentina.

Rodríguez M.C., Rosell P. 2005. Productividad y características fenológicas de los cultivares de papaya

Sunrise y Baixinho de Santa Amalia en invernadero de malla en la zona suroeste de la isla de Tenerife. Actas Portuguesas de Horticultura 6:245-249.

Samson, J.A. 1991. Papaya en Fruticultura Tropical. J.A. Samson (Eds.) Ed. Limusa Cap. 10: 305-319 - 396 pp.

Vázquez García, E.; Román Avendaño, E.E.; Ariza Flores, R. 2008. Fenología y Unidades Calor de Genotipos de papayo en el sur de Tamaulipas, México Rev. Fitotec. Mex. Vol. 31 (3): 45 - 48. 